Supporting Information

\title{
Investigations on the Fundamental Process of Cathode Electrolyte Interphase Formation and Evolution of High-Voltage Cathodes
}

Qinghao Lit ${ }^{1+}$, Yi Wang ${ }^{1,2,3 \dagger}$, Xuelong Wang ${ }^{1}$, Xiaorui Sun ${ }^{1,2}$, Jie-Nan Zhang ${ }^{l}$, Xiqian $Y u^{l, 2,4^{*}}$, Hong $\mathrm{Li}^{1,2,4^{*}}$

${ }^{1}$ Key Laboratory for Renewable Energy, Beijing Key Laboratory for New Energy Materials and Devices, Institute of Physics, Chinese Academy of Sciences, Beijing 100190, China

${ }^{2}$ College of Materials Sciences and Opto-Electronic Technology, University of Chinese Academy of Sciences, Beijing 100049, China

${ }^{3}$ Tianmu Lake Institute of Advanced Energy Storage Technologies Co. Ltd., Liyang 213300, China

${ }^{4}$ Yangtze River Delta Physics Research Center Co. Ltd., Liyang 213300, China

$\dagger$ These authors contributed equally.

*email: xyu@iphy.ac.cn; hli@iphy.ac.cn 
a) $L R-N C M$
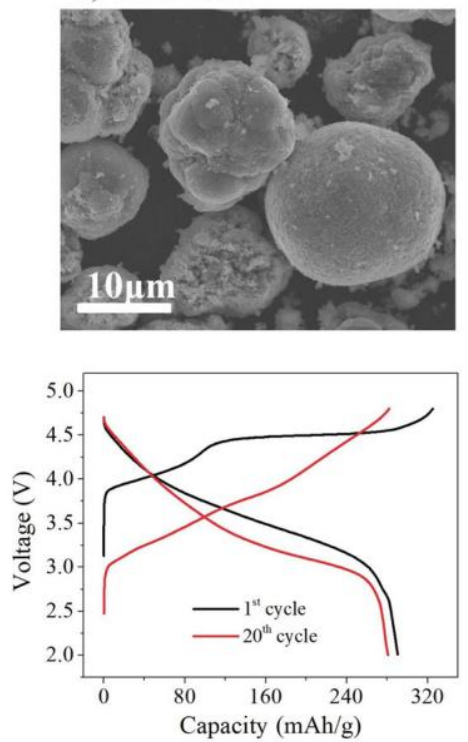

b) $L R M O$
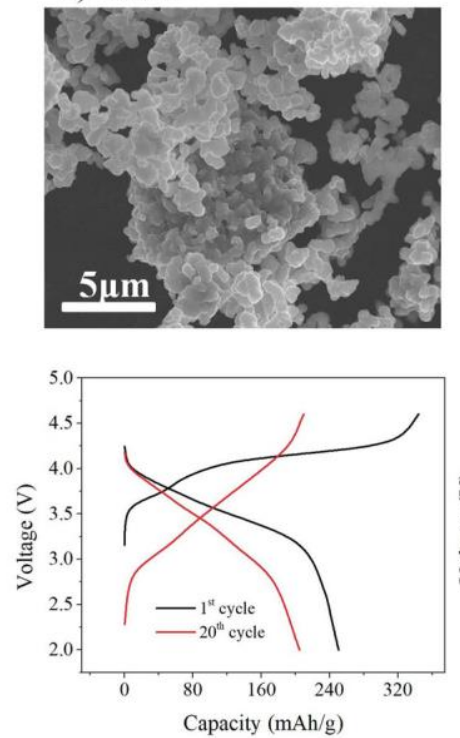

c) $L N M O$
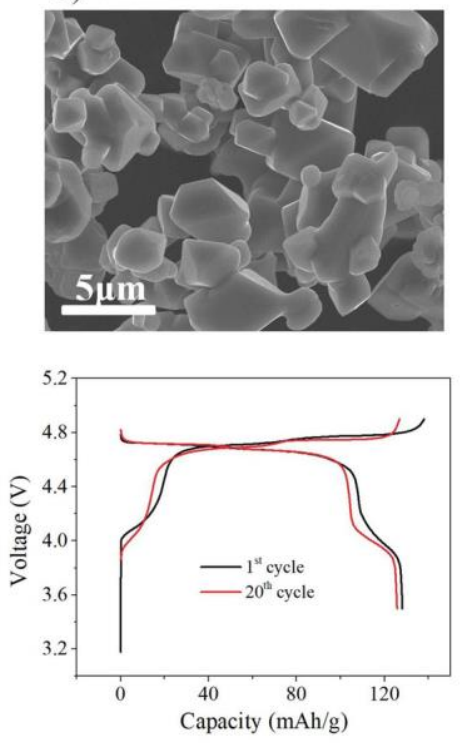

Figure S1 The SEM morphology of active materials of (a) layered $\mathrm{Li}_{1.144} \mathrm{Ni}_{0.136} \mathrm{Co}_{0.136} \mathrm{Mn}_{0.544} \mathrm{O}_{2}$ (LR-NCM), (b) layered $\mathrm{Li}_{2} \mathrm{Ru}_{0.5} \mathrm{Mn}_{0.5} \mathrm{O}_{3}$ (LRMO) and (c) spinel $\mathrm{LiNi}_{0.5} \mathrm{Mn}_{1.5} \mathrm{O}_{4}$ (LNMO). The voltage-capacity profiles of the three types of cathodes are shown correspondingly within their intrinsic voltage ranges, LR-NCM: 2.0-4.8 V, LRMO: 2.0-4.6 V, LNMO: 3.5-4.9 V, which demonstrates typical electrochemical behaviors of each cathode. 
(a) LR-NCM 4.8V-1D (3V)

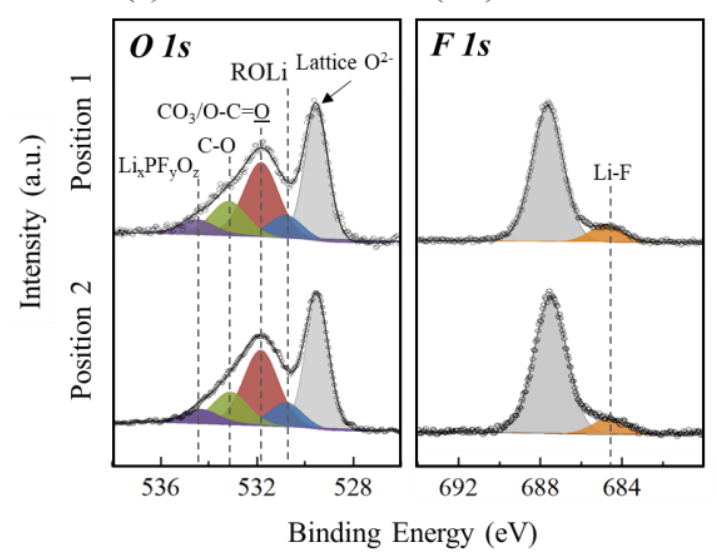

(b) NM 4.9V-1D (3.5V)

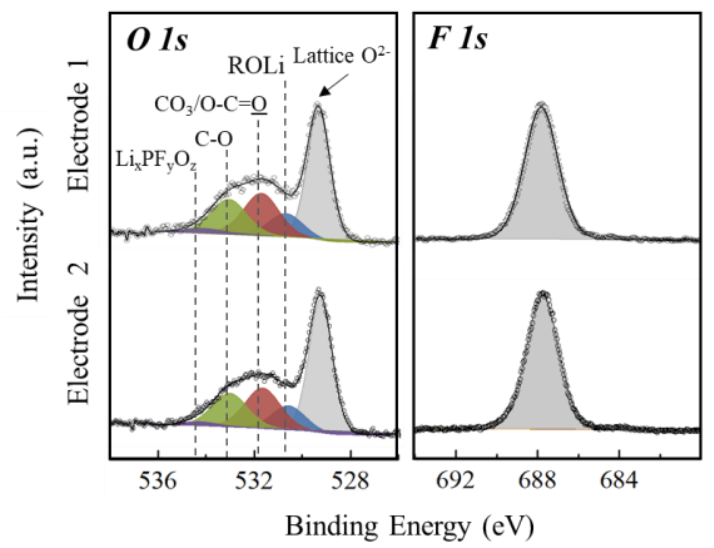

Figure S2 (a) O 1s and F 1s XPS collected on different sample positions on one discharged 3.0 V LR-NCM cathode after charge 4.8 V, which consist with each other. (b) O 1s and F 1s XPS collected on two separate samples of discharged 3.5 V LNMO after charge $4.9 \mathrm{~V}$, which overall consist with each other. 
a) $L R-N C M$

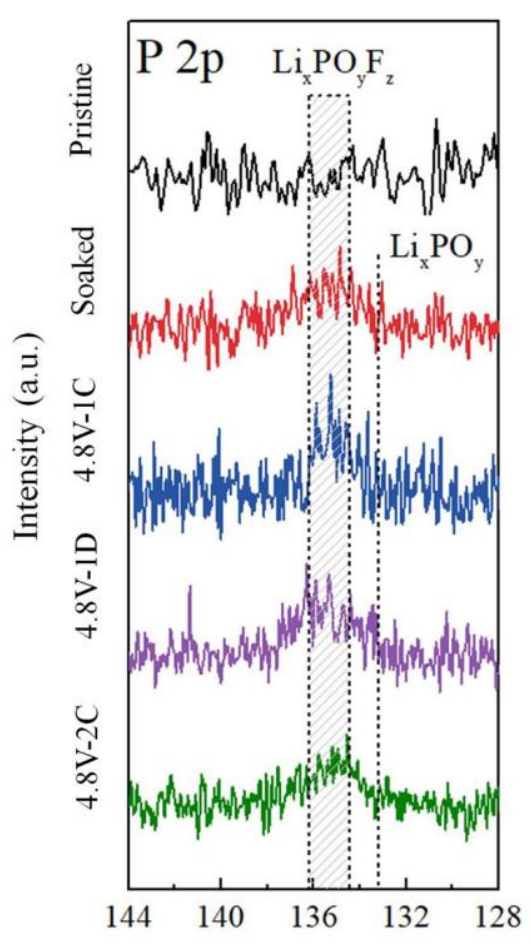

b) $L R M O$

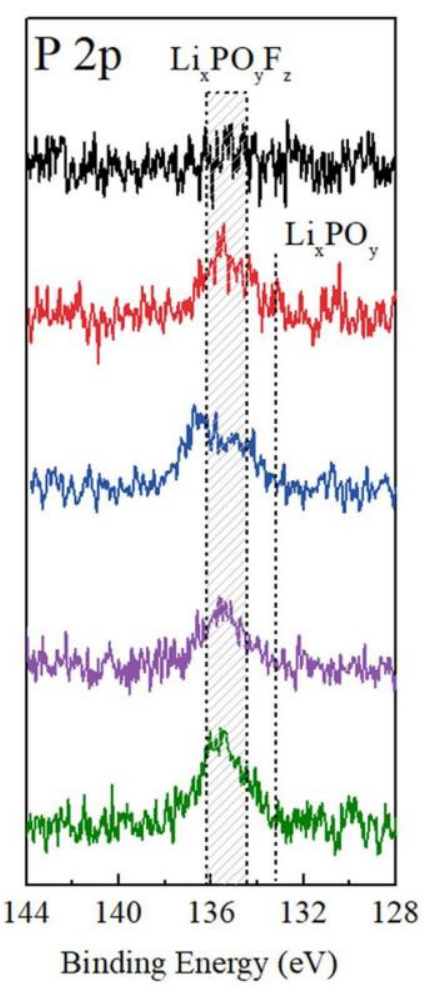

c) $L N M O$

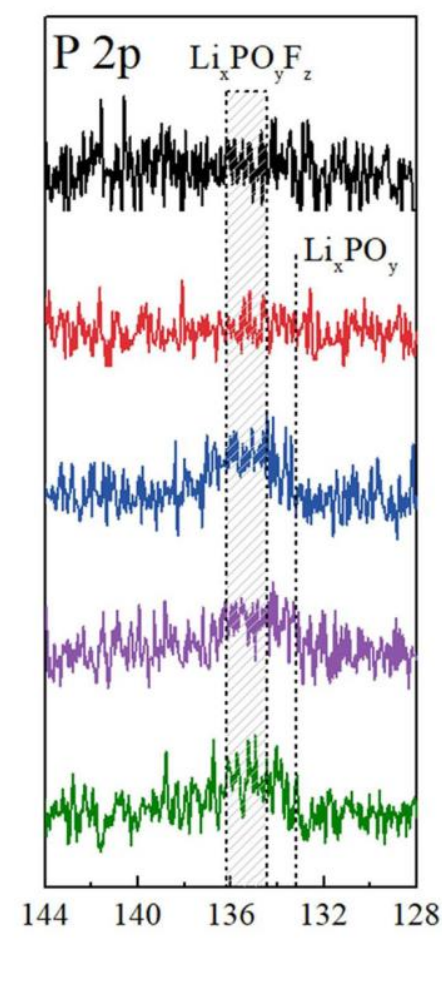

Figure S3 P 2p XPS spectra of (a) LR-NCM, (b) LRMO and (c) LNMO electrodes at different states. The pristine, electrolyte soaking for $24 \mathrm{~h}$, first charge $4.8 \mathrm{~V}$, first discharge $3 \mathrm{~V}$ and second charge $4.8 \mathrm{~V}$ states were arranged from top to bottom. $\mathrm{Li}$ salt decomposition induced $\mathrm{Li}_{x} \mathrm{PO}_{y}$ composition are highlighted 
a) charge

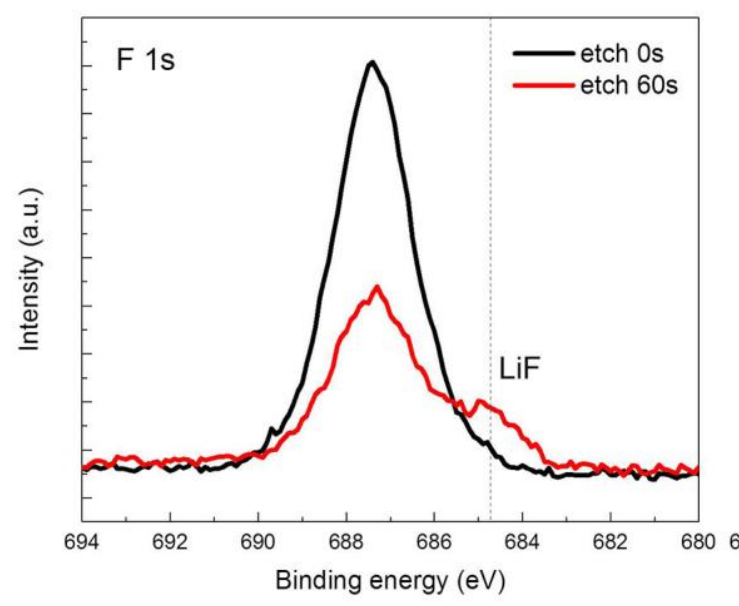

b) discharge

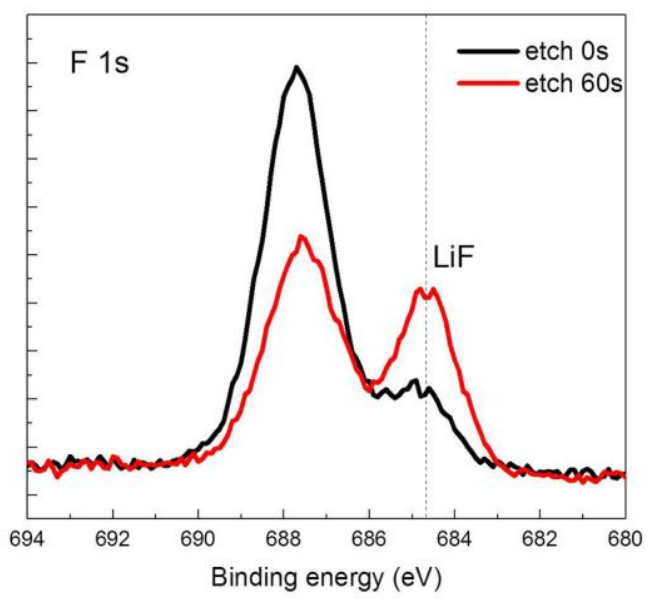

Figure S4 Comparison of F1s XPS spectra between fresh and etched cathode of LR-NCM at (a) charge and (b) discharge states. With $\mathrm{Ar}^{+}$etching for $60 \mathrm{~s}, \mathrm{LiF}$ related peak arising can be found obviously, implying inhomogeneous distribution of CEI components in depth direction. Discharged LR-NCM demonstrates higher LiF peak compared with charged LR-NCM with/without etching procedure. 

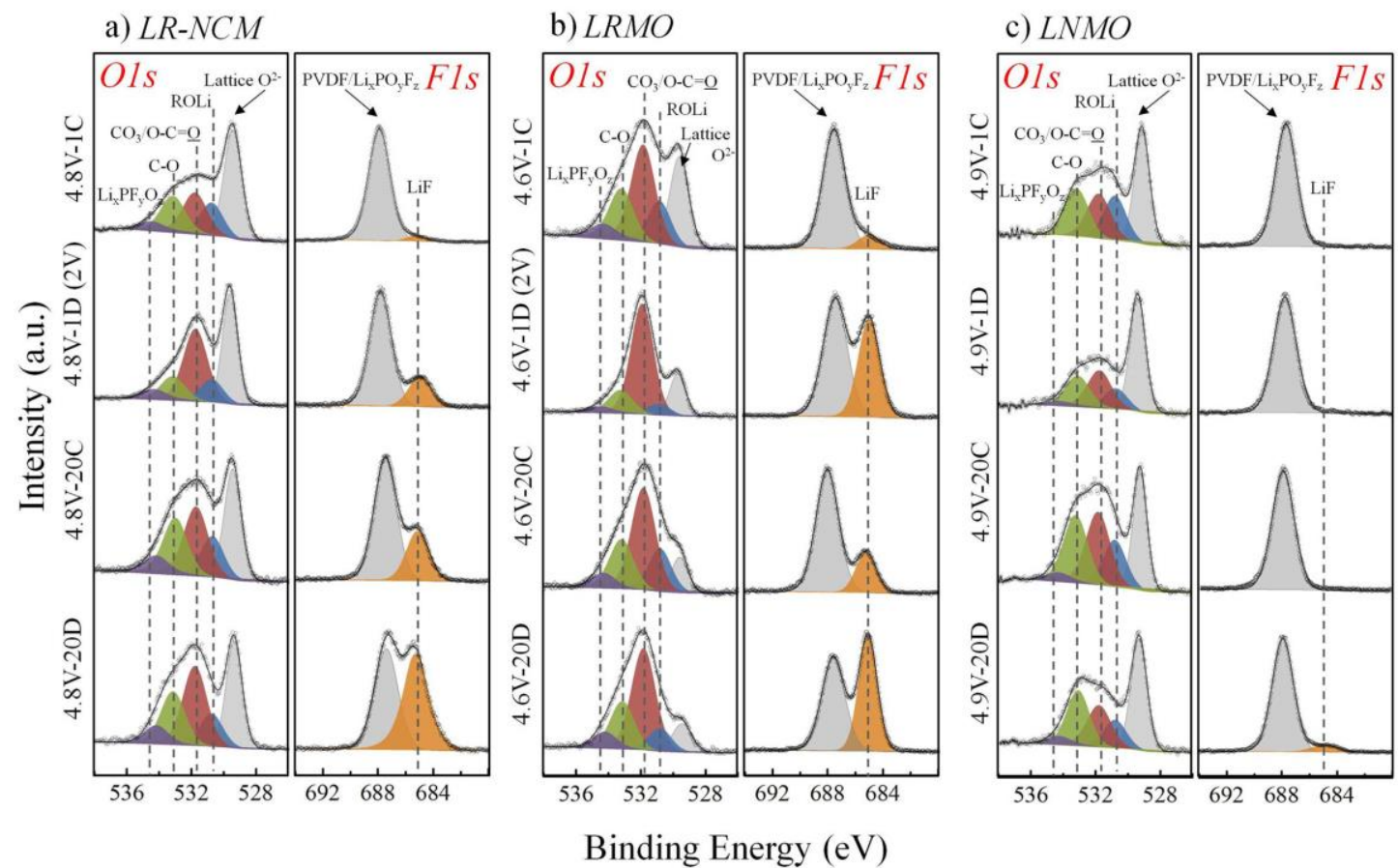

Figure S5 XPS spectra of the O 1s and F 1s collected from (a) LR-NCM, (b) LRMO and (c) LNMO electrodes cycled within their intrinsic voltage ranges (LR-NCM: 2.0-4.8 V, LRMO: 2.0-4.6 V, LNMO: 3.5-4.9 V). The XPS spectra of three types of cathodes in first cycle (charge and discharge states) and $20^{\text {th }}$ cycles (charge and discharge states) are included. 


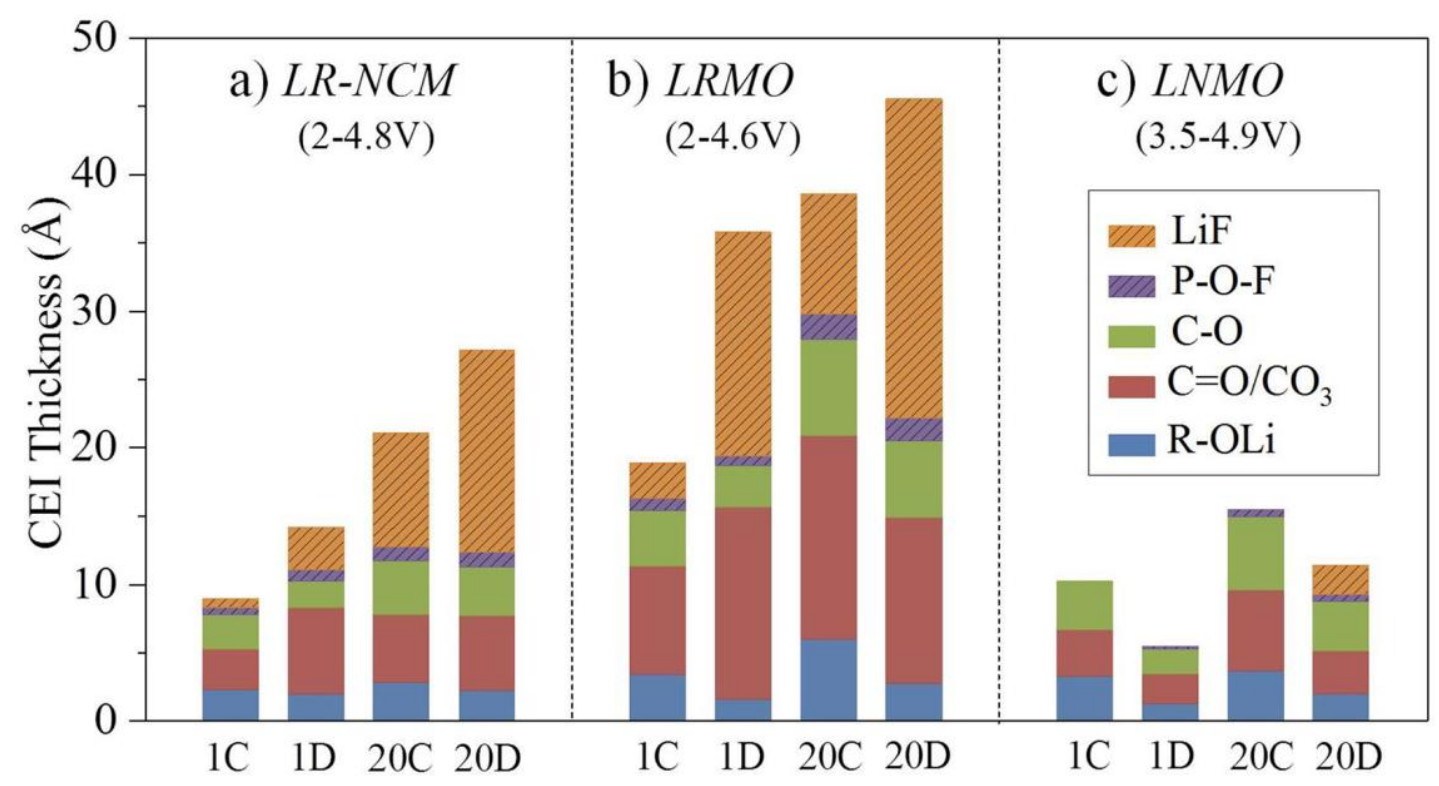

Figure S6 The comparison of the CEI thickness variation with long cycles among LR-NCM, LRMO and LNMO electrodes. Within each column, the contents of each decomposition products in $\mathrm{O} 1 \mathrm{~s}$ and $\mathrm{F} 1 \mathrm{~s}$ are given. Note that in $20^{\text {th }}$ cycles, the CEI thickness variations from charge to discharge states reproduce the trend in the first cycle in all three types of cathodes. Meanwhile, the CEI thickness gradually increases with long cycles. 

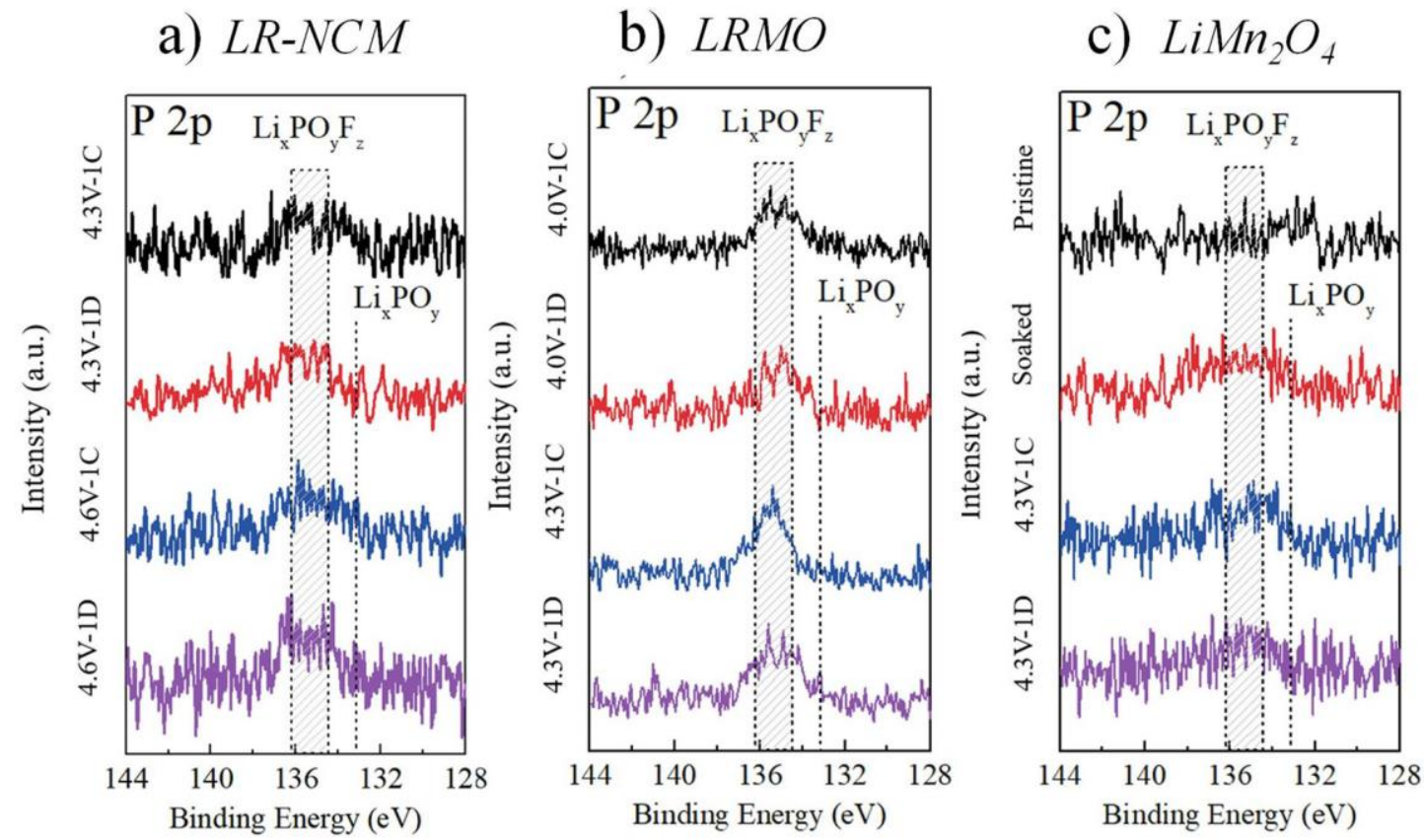

Figure S7 P 2p XPS spectra of (a) LR-NCM, (b) LRMO and (c) $\mathrm{LiMn}_{2} \mathrm{O}_{4}$ electrodes cycled at different voltage ranges. The comparison involves the influence of varying cut-off voltage and ARR (Anionic Redox Reaction) involving or not on CEI evolution behavior. 

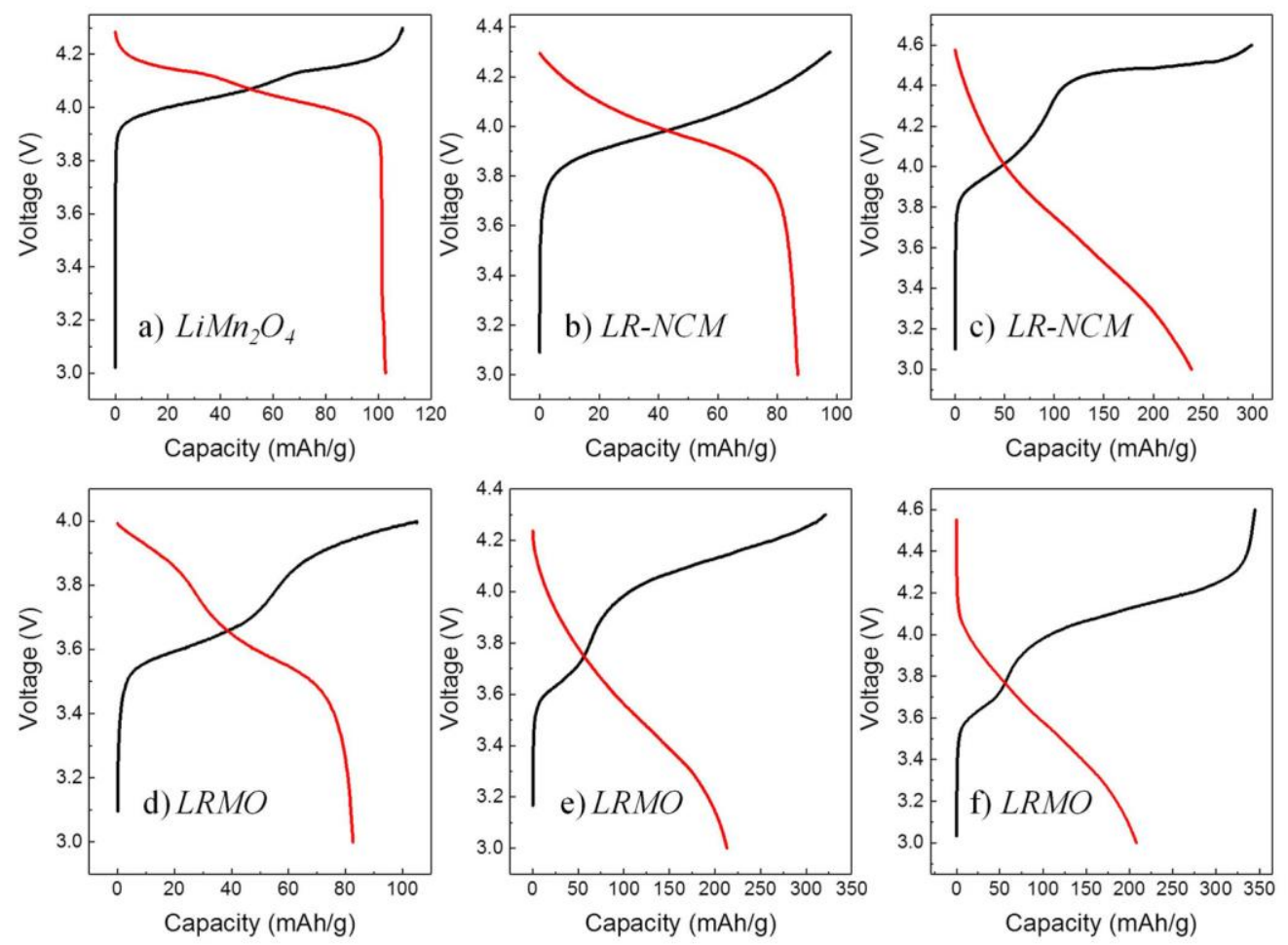

Figure S8 The voltage-capacity profiles of the three types of cathodes within varying cut-off voltages. (a) $\mathrm{LiMn}_{2} \mathrm{O}_{4}$ at 3.0-4.3 V, (b) LR-NCM at 3.0-4.3 V, (c) LR-NCM at 3.0-4.6 V, (d) LRMO at 3.0-4.0 V, (e) LRMO at 3.0-4.3 V and (f) LRMO at 3.0-4.6 V. 
Table S1 Quantitative analysis of O 1s and F 1s XPS spectra of LR-NCM, LRMO and LNMO electrodes at different states within 3.0-4.8 V. The peak assignments, BE (binding energy), FWHM (full width half maximum) and At\% (atomic ratio) are listed. The interphase species probed at pristine, electrolyte soaking for $24 \mathrm{~h}$, first charge $4.8 \mathrm{~V}$, first discharge $3 \mathrm{~V}$ and second charge $4.8 \mathrm{~V}$ states are tabulated.

\begin{tabular}{|c|c|c|c|c|c|c|c|c|c|c|c|c|c|c|c|c|c|}
\hline & \multirow[b]{2}{*}{ Peak } & \multirow[b]{2}{*}{ Assignments } & \multicolumn{3}{|l|}{ Pristine } & \multicolumn{3}{|l|}{ soaked } & \multicolumn{3}{|l|}{$4.8 \mathrm{~V}-1 \mathrm{C}$} & \multicolumn{3}{|c|}{$4.8 \mathrm{~V}-1 \mathrm{D}(3 \mathrm{~V})$} & \multicolumn{3}{|l|}{$4.8 \mathrm{~V}-2 \mathrm{C}$} \\
\hline & & & $\begin{array}{l}\text { BE } \\
(\mathrm{eV})\end{array}$ & $\begin{array}{l}\text { FWHM } \\
(\mathrm{eV})\end{array}$ & At\% & $\begin{array}{l}\mathrm{BE} \\
(\mathrm{eV})\end{array}$ & $\begin{array}{l}\text { FWHM } \\
(\mathrm{eV})\end{array}$ & At\% & $\begin{array}{l}\mathrm{BE} \\
(\mathrm{eV})\end{array}$ & $\begin{array}{l}\text { FWHM } \\
(\mathrm{eV})\end{array}$ & At\% & $\begin{array}{l}\text { BE } \\
(e V)\end{array}$ & $\begin{array}{l}\text { FWHM } \\
(\mathrm{eV})\end{array}$ & $A t \%$ & $\begin{array}{l}\text { BE } \\
(e V)\end{array}$ & $\begin{array}{l}\text { FWHM } \\
(\mathrm{eV})\end{array}$ & At\% \\
\hline \multirow{4}{*}{ LR-NCM } & \multirow{4}{*}{ O1s } & $\begin{array}{l}\text { Lattice } \\
\text { oxygen }\end{array}$ & 529.61 & 1.14 & 64.34 & 529.65 & 1.15 & 50.32 & 529.46 & 1.35 & 40.35 & 529.5 & 1.21 & 34.17 & 529.4 & 1.34 & 37.22 \\
\hline & & ROLi & 1 & 1 & 1 & 530.72 & 1.7 & 2.35 & 530.78 & 1.7 & 15.05 & 530.76 & 1.7 & 8.42 & 530.73 & 1.7 & 14 \\
\hline & & $\mathrm{C}=\mathrm{O} / \mathrm{CO}_{3}$ & 531.56 & 1.8 & 32 & 531.71 & 1.8 & 27.55 & 531.78 & 1.8 & 19.96 & 531.80 & 1.8 & 28.39 & 531.76 & 1.8 & 23.83 \\
\hline & & $\mathrm{C}-\mathrm{O}$ & 533.14 & 1.8 & 3.66 & 533.15 & 1.7 & 5.39 & 533.15 & 1.8 & 17.12 & 533.17 & 1.8 & 12.74 & 533.11 & 1.8 & 17.69 \\
\hline
\end{tabular}




\begin{tabular}{|c|c|c|c|c|c|c|c|c|c|c|c|c|c|c|c|c|c|}
\hline & & $\mathrm{Li}_{x} \mathrm{PFyO}_{z}$ & 1 & 1 & 1 & 534.29 & 1.7 & 1.86 & 534.42 & 1.7 & 3.56 & 534.43 & 1.7 & 4.97 & 534.41 & 1.7 & 3.94 \\
\hline & F1s & LiF & 1 & 1 & 1 & 685.05 & 2 & 12.53 & 685.18 & 1.89 & 3.96 & 684.76 & 2 & 11.31 & 684.89 & 1.88 & 3.32 \\
\hline \multirow{6}{*}{ LRMO } & \multirow{5}{*}{ 01s } & $\begin{array}{l}\text { Lattice } \\
\text { oxygen }\end{array}$ & 529.79 & 1.09 & 40.07 & 529.25 & 1.4 & 5.03 & 529.46 & 1.42 & 28.12 & 529.42 & 1.34 & 20.92 & 529.37 & 1.39 & 26.98 \\
\hline & & ROLi & 1 & 1 & 1 & 530.65 & 1.7 & 6.75 & 530.78 & 1.7 & 17.84 & 530.73 & 1.7 & 15.47 & 530.74 & 1.7 & 19.54 \\
\hline & & $\mathrm{C}=\mathrm{O} / \mathrm{CO}_{3}$ & 531.60 & 1.89 & 38.4 & 531.86 & 1.8 & 18.92 & 531.8 & 1.8 & 30.08 & 531.74 & 1.8 & 36.03 & 531.74 & 1.8 & 28.16 \\
\hline & & $\mathrm{C}-\mathrm{O}$ & 533.27 & 2.01 & 2.87 & 533.15 & 1.8 & 4.28 & 533.12 & 1.8 & 18.23 & 533.14 & 1.8 & 10.38 & 533.11 & 1.8 & 17.94 \\
\hline & & $\mathrm{Li}_{x} \mathrm{PFyO}_{z}$ & 1 & 1 & 1 & 534.31 & 1.7 & 1.03 & 534.41 & 1.7 & 3.15 & 534.20 & 1.7 & 2.65 & 534.21 & 1.7 & 3.45 \\
\hline & F1s & LiF & 684.69 & 1.73 & 18.67 & 684.82 & 1.84 & 63.99 & 685.03 & 1.81 & 2.58 & 684.65 & 1.84 & 14.55 & 684.88 & 2.01 & 3.93 \\
\hline \multirow[t]{2}{*}{ LNMO } & \multirow[t]{2}{*}{ 01s } & $\begin{array}{l}\text { Lattice } \\
\text { oxygen }\end{array}$ & 529.75 & 1.09 & 60.36 & 529.65 & 1.11 & 49.51 & 529.59 & 1.13 & 39.04 & 529.56 & 1.13 & 43.7 & 529.43 & 1.13 & 36.54 \\
\hline & & ROLi & 1 & 1 & 1 & 530.65 & 1.7 & 3.97 & 530.88 & 1.7 & 14.97 & 530.85 & 1.7 & 13.98 & 530.74 & 1.7 & 15.6 \\
\hline
\end{tabular}




\begin{tabular}{|l|l|l|l|l|l|l|l|l|l|l|l|l|l|l|l|l|l|l|l|}
\hline & $\mathrm{C}=\mathrm{O} / \mathrm{CO}_{3}$ & 531.72 & 1.86 & 33.78 & 531.78 & 1.85 & 29.97 & 531.88 & 1.8 & 23.98 & 531.86 & 1.8 & 17.65 & 531.77 & 1.8 & 24.38 \\
$\mathrm{C}-\mathrm{O}$ & 533.33 & 1.8 & 5.86 & 533.36 & 1.7 & 6.89 & 533.28 & 1.8 & 18.36 & 533.24 & 1.8 & 12.16 & 533.15 & 1.8 & 19.79 \\
& $\mathrm{Li}_{x} \mathrm{PFyO}_{2}$ & $\backslash$ & $\backslash$ & $\backslash$ & $\backslash$ & $\backslash$ & $\backslash$ & 534.50 & 1.7 & 3.65 & 534.55 & 1.7 & 3.08 & 534.48 & 1.7 & 3.69 & \\
\hline
\end{tabular}


Table S2 Quantitative analysis of O 1s and F 1s XPS spectra of LR-NCM, LRMO and LNMO electrodes at different cycle states within their intrinsic voltage ranges. The peak assignments, BE, FWHM and At\% are listed. The interphase species probed at first cycle (charge and discharge states) and $20^{\text {th }}$ cycles (charge and discharge states) are tabulated.

\begin{tabular}{|c|c|c|c|c|c|c|c|c|c|c|c|c|c|c|}
\hline & \multirow[b]{2}{*}{ Peak } & \multirow[b]{2}{*}{ Assignments } & \multicolumn{3}{|l|}{$4.8 \mathrm{~V}-1 \mathrm{C}$} & \multicolumn{3}{|c|}{$4.8 \mathrm{~V}-1 \mathrm{D}(2 \mathrm{~V})$} & \multicolumn{3}{|c|}{$2-4.8 V-20 C$} & \multicolumn{3}{|c|}{$4.8 \mathrm{~V}-20 \mathrm{D}(2 \mathrm{~V})$} \\
\hline & & & $\mathrm{BE}(\mathrm{eV})$ & $\begin{array}{l}\text { FWHM } \\
(\mathrm{eV})\end{array}$ & At\% & $\mathrm{BE}(\mathrm{eV})$ & $\begin{array}{l}\text { FWHM } \\
(\mathrm{eV})\end{array}$ & At\% & $\mathrm{BE}(\mathrm{eV})$ & $\begin{array}{l}\text { FWHM } \\
(\mathrm{eV})\end{array}$ & At\% & $\mathrm{BE}(\mathrm{eV})$ & $\begin{array}{l}\text { FWHM } \\
(\mathrm{eV})\end{array}$ & At\% \\
\hline \multirow{3}{*}{ LR-NCM } & \multirow{3}{*}{ O1s } & $\begin{array}{l}\text { Lattice } \\
\text { oxygen }\end{array}$ & 529.46 & 1.35 & 40.35 & 529.59 & 1.15 & 30.66 & 529.49 & 1.28 & 21.29 & 529.41 & $\mid 1.21$ & 15.49 \\
\hline & & $\begin{array}{l}\mathrm{ROLi} \\
\mathrm{C}=\mathrm{O} / \mathrm{CO}_{3}\end{array}$ & $\begin{array}{l}530.78 \\
531.78\end{array}$ & $\begin{array}{l}1.7 \\
1.8\end{array}$ & $\begin{array}{l}15.05 \\
19.96\end{array}$ & $\begin{array}{l}530.72 \\
531.75\end{array}$ & $\begin{array}{l}1.75 \\
1.8\end{array}$ & $\begin{array}{l}9.34 \\
31.09\end{array}$ & $\begin{array}{l}530.75 \\
531.75\end{array}$ & $\begin{array}{l}1.7 \\
1.8\end{array}$ & $\begin{array}{l}10.41 \\
18.48\end{array}$ & $\begin{array}{l}530.82 \\
531.83\end{array}$ & $\begin{array}{l}1.7 \\
1.8\end{array}$ & $\begin{array}{l}6.72 \\
17.02\end{array}$ \\
\hline & & $\mathrm{C}-\mathrm{O}$ & 533.15 & 1.8 & 17.12 & 533.16 & 1.8 & 9.83 & 533.09 & 1.8 & 14.7 & 533.19 & 1.8 & 11.19 \\
\hline
\end{tabular}




\begin{tabular}{|c|c|c|c|c|c|c|c|c|c|c|c|c|c|c|}
\hline & F1s & LiF & 685.18 & 1.89 & 3.96 & 685.03 & 2.20 & 15.09 & 685.11 & 2 & 31.17 & 684.89 & 1.98 & 46.08 \\
\hline & & & $4.6 \mathrm{~V}-1 \mathrm{C}$ & & & 4.6V-1D & & & $4.6 \mathrm{~V}-20 \mathrm{C}$ & & & $4.6 \mathrm{~V}-20$ & (2V) & \\
\hline \multirow{4}{*}{ LRMO } & \multirow{3}{*}{ 01s } & $\begin{array}{l}\text { Lattice } \\
\text { oxygen }\end{array}$ & 529.54 & 1.42 & 23.95 & 529.65 & 1.22 & 9.82 & 529.65 & 1.24 & 8.48 & 529.47 & 1.49 & 5.89 \\
\hline & & C-O & 533.16 & 1.8 & 16.27 & 533.22 & 1.8 & 7.57 & 533.25 & 1.8 & 16.71 & 533.16 & 1.8 & 11.56 \\
\hline & & $\mathrm{Li}_{x} \mathrm{PFyO}_{z}$ & 534.35 & 1.7 & 3.76 & 534.44 & 1.7 & 1.81 & 534.37 & 1.7 & 4.47 & 534.29 & 1.7 & 3.6 \\
\hline & F1s & LiF & 685.01 & 2 & 10.22 & 684.91 & 1.89 & 41.35 & 685.18 & 1.77 & 20.86 & 685.11 & 2.03 & 48.23 \\
\hline
\end{tabular}




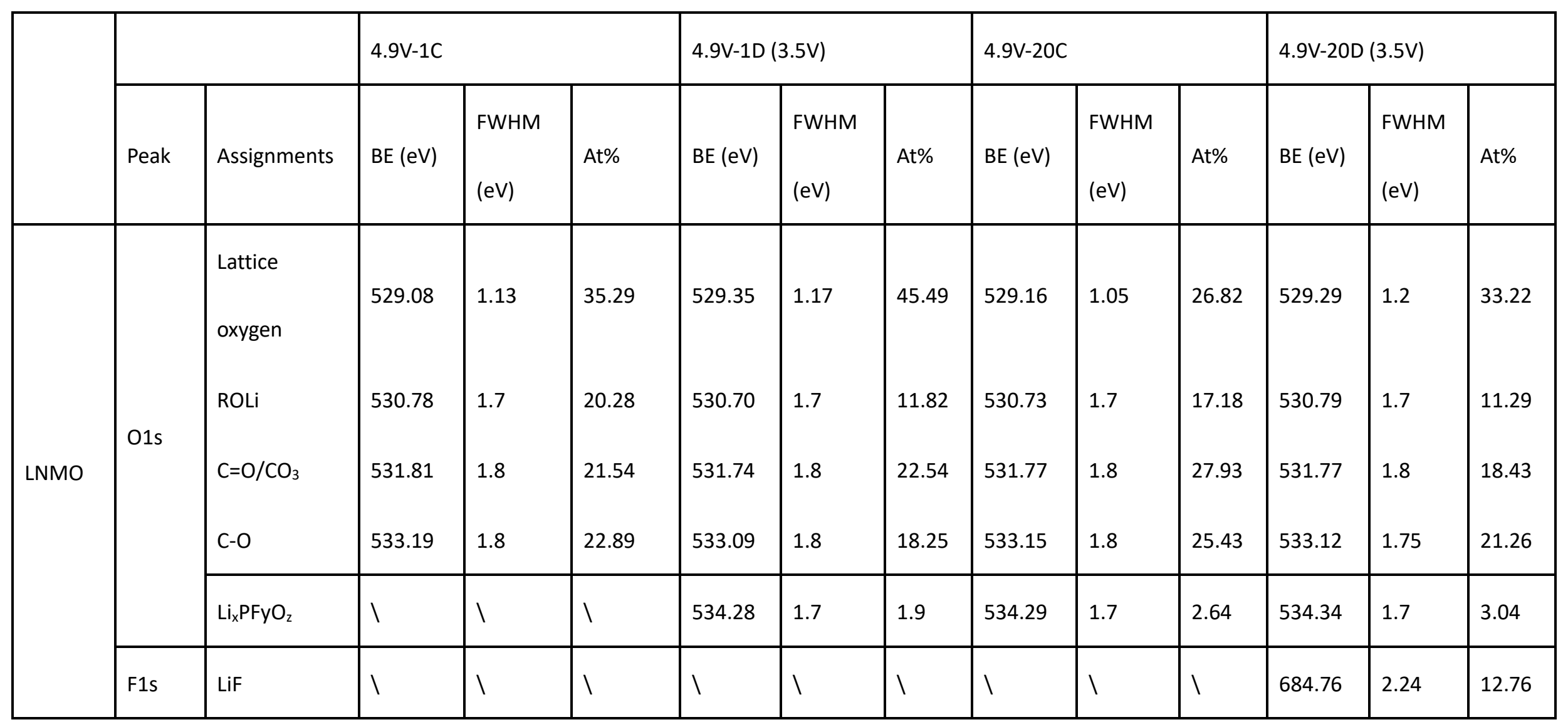


Table S3 Quantitative analysis of O 1s and F 1s XPS spectra of LR-NCM, LRMO and $\mathrm{LiMn}_{2} \mathrm{O}_{4}$ electrodes at different states within varying voltage ranges. The peak assignments, BE, FWHM and At\% are listed. The interphase species probed at varying cut-off voltage and ARR

(Anionic Redox Reaction) involving or not are tabulated.

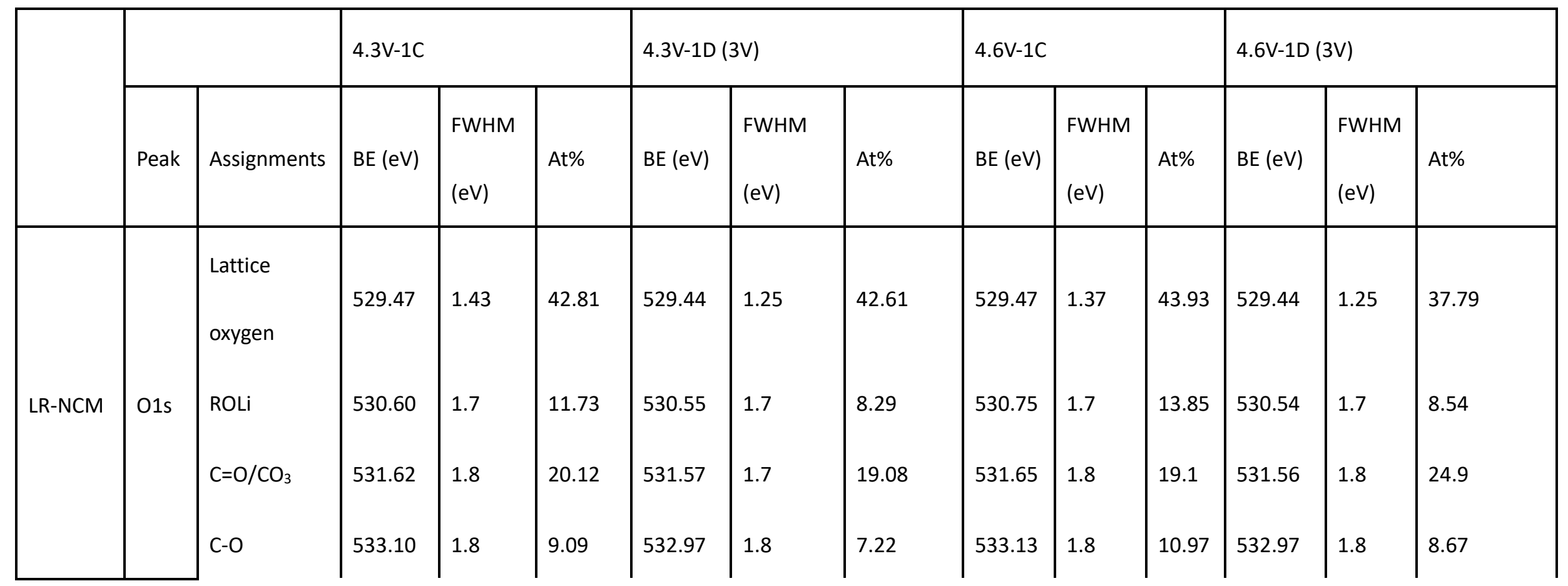




\begin{tabular}{|c|c|c|c|c|c|c|c|c|c|c|c|c|c|c|}
\hline & F1s & LiF & 685.15 & 2.41 & 14.49 & 685.13 & 1.88 & 21.39 & 685.07 & 2.2 & 9.67 & 684.97 & 2.03 & 17.83 \\
\hline & & & 4.0V-1C & & & 4.0V-1D & & & $4.3 \mathrm{~V}-1 \mathrm{C}$ & & & $4.3 \mathrm{~V}-1 \mathrm{D}$ & $3 V)$ & \\
\hline \multirow{4}{*}{ LRMO } & \multirow{3}{*}{ 01s } & $\begin{array}{l}\text { Lattice } \\
\text { oxygen }\end{array}$ & 529.37 & 1.16 & $\mid \begin{array}{l}\mid 10.8 \\
\end{array}$ & 529.44 & 1.17 & 20.1 & 529.46 & 1.20 & 10.74 & 529.37 & 1.32 & 6.49 \\
\hline & & $\mathrm{C}-\mathrm{O}$ & 533.21 & 1.8 & 8.07 & 533.18 & 1.8 & 6.89 & 533.14 & 1.8 & 11.02 & 533.22 & 1.8 & 5.3 \\
\hline & & $\mathrm{Li}_{x} \mathrm{PFyO}_{z}$ & 534.42 & 1.7 & \begin{tabular}{|l|}
1.97 \\
\end{tabular} & 533.43 & 1.7 & 1.85 & 534.45 & 1.7 & 3.03 & 534.36 & 1.7 & 2.09 \\
\hline & F1s & LiF & 685.15 & 1.66 & 33.5 & 685.17 & 1.79 & 13.45 & 685.19 & 1.71 & 20.43 & 685.07 & 1.58 & 42.13 \\
\hline
\end{tabular}




\begin{tabular}{|c|c|c|c|c|c|c|c|c|c|c|c|c|c|c|}
\hline & Peak & Assignments & $B E(e V)$ & $\begin{array}{l}\text { FWHM } \\
(\mathrm{eV})\end{array}$ & At\% & $\mathrm{BE}(\mathrm{eV})$ & $\begin{array}{l}\text { FWHM } \\
(\mathrm{eV})\end{array}$ & At\% & $B E(e V)$ & $\begin{array}{l}\text { FWHM } \\
(\mathrm{eV})\end{array}$ & At\% & $\mathrm{BE}(\mathrm{eV})$ & $\begin{array}{l}\text { FWHM } \\
(\mathrm{eV})\end{array}$ & At\% \\
\hline \multirow{3}{*}{$\mathrm{LiMn}_{2} \mathrm{O}_{4}$} & \multirow[t]{2}{*}{ 01s } & ROLi & 1 & 1 & 1 & 530.69 & 1.7 & 4.74 & 530.61 & 1.7 & 13.5 & 530.56 & 1.7 & 13.34 \\
\hline & & $\mathrm{C}=\mathrm{O} / \mathrm{CO}_{3}$ & 531.50 & 1.89 & 32.97 & 531.66 & 1.8 & 28.06 & 531.59 & 1.8 & 19.27 & 531.56 & 1.8 & 17.77 \\
\hline & F1s & LiF & 1 & 1 & 1 & 684.74 & 1.73 & 8.21 & 684.77 & 1.82 & 3.36 & 684.68 & 2.02 & 6.82 \\
\hline
\end{tabular}

